\title{
Sales Practices: Monitoring Sales Activity for Anomalous Behaviors
}

\author{
Dr. Jimmie Lenz* \\ USA
}

*Corresponding Author: Dr. Jimmie Lenz, USA

\begin{abstract}
The use of sales incentives (commissions, bonuses, etc.) to motivate the behavior of salespeople has a long history, as does the negative effect on customers that sometimes results. This mistreatment is sometimes considered the "cost of doing business" but recent cases of unchecked and large-scale customer abuse have focused particular attention on the financial services industry and what can be done to detect this behavior. We have developed a methodology to detect both customer sales and individual product behaviors that are indicative of problematic situations that require additional examination. Our methodology goes beyond the aggregate sales, which are primarily discussed in the literature, to highlight individuals and/or groups that are often obviated when analyzing such data.
\end{abstract}

\section{BACKGROUND}

The use of incentives to influence the behavior of sales people has a long history in the United States. This reward system came about in the late nineteenth century, and was widely popularized by the National Cash Register (NCR) Company. In the early years the company's motivation was due to a lack of cash to pay salaries, resulting in NCR paying as much as $50 \%$ in commissions, and illustrated the effect of changing commissions on behavior. As time went on the notion of utilizing Short Term Incentives (STI) became ubiquitous in all types of sales programs, and it continues to be used to motivate certain behaviors that align the agent and principal goals. The ability to affect behavior is well documented (Thaler and Sunstein 2008), however, there is a plethora of research on the unintended effects that can result from these programs. Of particular interest is the understanding by various researchers that STIs, if left unchecked, can lead to unethical and non-customer oriented behavior, among other things (Zoltners, Sinha, and Lorimer 2013).

Normally an analysis that seeks to discover anomalies, even in a dynamic environment, relies significantly on established (i.e. proven) benchmarks of some type. However, sales organizations, whether related to new or existing products/services, have been challenged to develop such accurate benchmarks or "forecasts." In many cases "forecasts" have little, if any, basis and reflect the wish of finance or management. This leads to incentive plans that are not only unrealistic given a particular environment, but also encouraging of behavior that is the antithesis of client centric. Organizations that utilize blanket sales goals across large geographic and/or client segments, irrespective of the nuances of the various segments, exhibit this flawed thinking. A variety of methods have been employed and discussed in the literature, including those that incorporate the salespeople themselves (White 1984), which raise questions about the inherent principal agent conflict. In the best case, accurate forecasts may benefit the corporation, but may not benefit its customers.

While there are a number of examples of the problematic application of STIs in the current environment this is not a new phenomenon. In the early 1990's Sears, Roebuck and Co. incentivized sales people in its auto division with a straight commission payout resulting in unnecessary work being performed and Sears paying fines and compensation (Ganzel 1998). In more recent times there have been other types of sales-based issues that have resulted in industry-wide settlements. The Auction Rate Securities settlements resulted in billions of dollars in repurchases and fines being paid by many of the world's leading financial services firms. In both of these cases and most others there were signs that something was amiss, so why were these issues not identified? There appear to be several reasons: 1) there is a hesitancy by management to look (what appears to be) a gift horse in the mouth; 2) few firms have a solid scientific basis for forecasting sales; and 3) there is little understanding of how a detection measurement might be accomplished. 


\subsection{Current Environment}

There is no shortage of current examples of this problematic behavior, and in some cases it is more basic for individuals covered by sales plans than gains resulting from commissions and bonuses. The achievement of sales thresholds (i.e. goals, targets, etc.) utilized by some organizations are often the basis of retaining an individual's job. Although the research is a bit less available on this aspect, I would expect that maintaining employment would likely prove, in most cases, to be more of an incentive to engage in nefarious behavior than a bonus or commission (Kahneman and Tversky 1979). While such noted researchers as W. Edward Deming (1986) and Alfie Kohn (1993) have found that incentives are poor motivators and create no lasting commitment, this structure endures. While the tenure and belief in paying salespeople according to these types of incentive programs is not likely to change radically, to ensure customers receive appropriate products and services there is a need to dynamically measure and understand the product sales behaviors being motivated by these programs.

A timely illustration is provided in the Independent Directors of the Board of Wells Fargo regarding sales practices (i.e. the fake accounts). As the report highlights, "This was especially true in areas where bad practices tended to disproportionately cluster, like Los Angeles and Arizona." It illustrates that there were clustering events that might have been detected through the right type of objective and independent analysis. The report also profiles one senior manager who moved from Los Angeles to Florida, replicating the same growth of low quality accounts. One would expect this to be a very detectable pattern. However, as mentioned previously, there may be different reasons for not catching this behavior. For firms that rely on staff or culture to identify and report such issues, there is an additional passage in the report that "managers also explicitly encouraged their subordinates to sell unnecessary products," This indicates that confidence in management to detect such issues may be misplaced.

\section{Methodology}

The development of a scientifically-based sales measurement methodology is the purpose of this paper. While other authors have attempted a similar goal, they have often relied on user-defined thresholds (Hao, Dayal and Keim 2009). What we have detailed is a methodology that utilizes historic observations and external variables to understand the expected behavior in the environment in which an activity occurs. That is, this is not a simple linear path for each sales person, group, or office (from here on referred to in general terms as a "unit') but is instead a formulaic approach to each unit's unique expected performance. There is a mistaken belief that historic demand drivers continue in perpetuity (Barnett 1988). The advent of machine learning and other techniques further our ability to leverage this paradigm. The value, of course, is that once an individual unit's expected performance is well understood, anomalies will become apparent and can be investigated. The research has revealed that such an approach can not only focus on anomalous unit behavior, but also product behavior, as in the case of Auction Rate Securities mentioned previously.

Given the examples provided in the referenced report of the Independent Directors of the Board of Wells Fargo, the ability to assess the environment quantitatively would seem to provide a number of opportunities. It is necessary to keep in mind that this is but one example; there is no shortage from which to draw from. A review of the information available provides a good basis from which to understand the value of detection methodologies that could be explored and tested, along with a number of guidelines.

The ability of practitioners to develop reliable predictions based on that impact sales demand is well documented (Chase 1993). Data concerning all facets of sales and the resulting experience (e.g. the decrease in quality accounts in the case of Wells Fargo) is necessary to have in a format that can be analyzed. These, of course, will be used along with the dependent variable to understand which are the significant variables. Additionally, external data may be utilized (e.g. macro or macroeconomic information) to facilitate aspects of the analysis to account for trends that may affect sub-populations and thus be expected to affect less than the whole population. As stated there is a need to understand the environment in which an event occurs so that the analysis necessary to detect such issues must be reflective of the current environment and utilize historical context to detect possible issues while at the same time minimizing false positives.

Data regarding specific product sales is understandably limited, thus making specific research a bit challenging. However, given the papers abstract, detection of anomalous events, there are other 
populations that present similar opportunities to illustrate the value available. A population that offers millions of data points across a large and distributed population (not to mention a plethora of research), U.S. mortgages, was chosen to illustrate the methodology.

The findings related to the U.S. mortgage market, specifically related to types of mortgages (i.e. fixed-rate and adjustable-rate) provide a good basis from which to illustrate the methodology. As illustrated (Campbell and Cocco 2014), both adjustable-rate and fixed-rate mortgages have similar default rates. This is a bit unintuitive given the press related to adjustable mortgages. However, beyond the shared sensitivity to home prices, the explanatory variable affecting defaults are quite different. The adjustable-rate mortgages defaults are more likely to increase when both interest rates and inflation increase, which results in higher payments. Fixed-rate mortgages, on the other hand, experience higher default rates when inflation and interest rates decrease. This understanding of behaviors allowed for an understanding of what is expected in a given environment.

To better illustrate the chart below displays the relationship between delinquencies and the 10 -year U.S. Treasury yield from 1991-2017. The Delinquency Rate is comprised of loans secured by real estate, by all commercial banks on a percentage basis as reported quarterly on a seasonally adjusted basis in the Federal Reserve Economic Data.

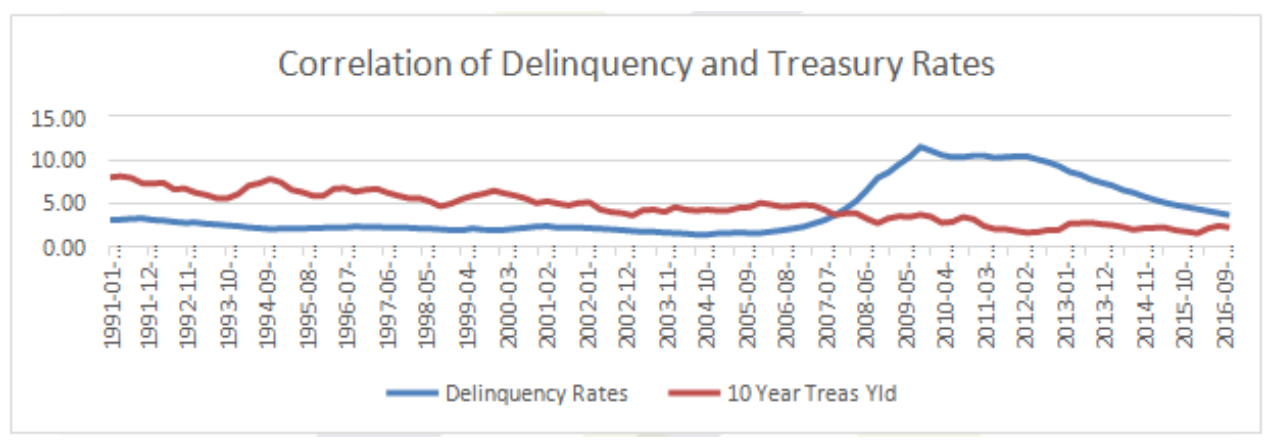

To provide additional perspective on the chart, when the U.S. Treasury is lagged by 6 months, and both values are logged, the correlation from 1991-2006 is .89. Clearly the historic correlation breaks, which is of course the anomalous behavior of interest. The regression statistics for the same period are:

\begin{tabular}{|l|l|}
\hline \multicolumn{2}{|c|}{ Regression Statistics } \\
\hline Multiple R & 0.784737 \\
\hline R Square & 0.615813 \\
\hline Adjusted R Square & 0.609189 \\
\hline Standard Error & 0.100352 \\
\hline Observations & 60 \\
\hline
\end{tabular}

These regression statistics have a P-Value of 1.18E-13.

We use the delinquency rates to illustrate the overall impact. However, when mortgages are isolated by type, the performance of the adjustable-rate products becomes that much more pronounced.

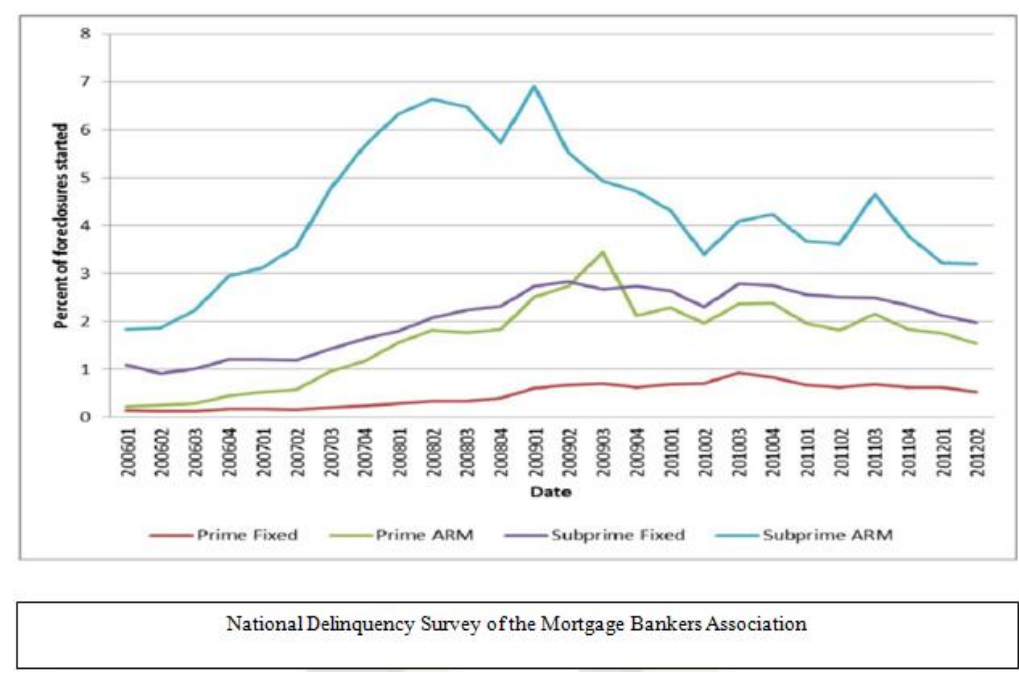


Given the research findings and the interest rate environment (a general decline), the realized behavior deviated significantly from the expected performance. The adjustable-rate mortgages experienced a much higher rate of defaults than forecast, given the interest and inflation rate environment. This deviation led to additional research and to the conclusion that borrowers with riskier income streams, possibly correlated positively to interest rates, had utilized adjustable-rate loans (Campbell and Cocco 2014). This conclusion of increased market participation by increasingly risky borrowers also seems substantiated by the significant growth in mortgage balances, an increase exceeding $50 \%$ in 5 years. Lastly the researchers find that that "teaser rates" and other schemes to defer principal payments tend to increase default rates.

The example drawn from the U.S. mortgage market provides context for the approach and methodology, while the deviation from the expected noted in 2006 mortgage balances continued to grow for almost 2 years. At this point in 2008 about 1 in 20 mortgages were delinquent, and of course this would rise to greater than 1 in 10 within a short period of time. Had the explanatory variables been discovered, well understood, and modeled appropriately, when the higher than expected defaults were initially realized, there would have been immediate realization that some anomalous event had had an effect on the population. Even without the cognizance of the significant balance growth and the questionable payment deferral practices, detection of the unexpected default growth would have provided reason for examination. With this knowledge proactive actions could have been taken to assess the impact and address the issue.

\section{CONCLUSION}

The ability to monitor the appropriateness of sales made by groups or individuals incented for such activity is challenging given the inherent conflicts that exist, but is necessary and receiving regulatory scrutiny. Given the fact that incentives come in a variety of forms besides direct monetary compensation, including but not limited to promotion opportunities, special recognition events, and continued employment control via compensation may not achieve the desired results. Although institutions attempt to control behavior through compensation reviews for conflicts, and/or culture, the fact remains that these conflicts are inherent to the sales process. While there is no doubt that incentives can be utilized to modify behavior that enhances sales, there is an optimal point after which consequences will be realized.

The value of modeling expected behavior, as illustrated in the mortgage example, is a methodology that takes into account the unique attributes of a product and the dynamics of the environment. The primary steps in constructing models to detect possible problems include the discernment and understanding of the significant explanatory variables that affect the sales of new and existing products. These variables, and the relative weightings, are discovered through established processes which may include machine learning or other automated techniques that allow for a depth of discovery previously limited by the available techniques. The variables discovered can be used in the model to forecast expected sales relative to the environment in which they occur, and alert the model owner when investigation into certain practices may be warranted. Additionally, such modeling allows the user to understand probable behavior based on various scenarios that can be facilitated through the methods.

\section{REFERENCES}

[1] Campbell, John and Cocco, Joao (2014), "The Model of Mortgage Default"

[2] Chase, Charles (1993), "Ways to Improve Sales Forecasts" The Journal of Business Forecasting Methods \& Systems vol. 12, No. 3

[3] Deming, W.E. (1986), Out of the Crisis: Quality, Productivity, and Competitive Position, Cambridge: Cambridge University Press

[4] Hao, M., Dayal, U., and Keim, D. (2009), "Visual Analytics of Anomaly Detection in Large Data Streams" Ganzel, R. (1998), "What's Wrong with Pay for Performance?" Training, 35 (12), 34-40

[5] Kahneman, D. and Tversky, A. (1979), Prospect Theory: An Analysis of Decision under Risk, Econometrica, 47 (2), pp.263-291, March 1979

[6] Kohn, A., (1993), "Why Incentives Plans Cannot Work" Harvard Business Review, 71 (5), 54-63

[7] Thaler, R. and Sunstein, C. (2008), "Nudge", Penguin Books 
[8] Zoltners, A., Sinha, P., Lorimer, S. (2013) "Breaking the Sales Force Incentive Addiction: A Balanced Approach to Sales Force Effectiveness" Journal of Personal Selling and Sales Management, 32(2):171186

\section{AUTHOR'S BIOGRAPHIES}

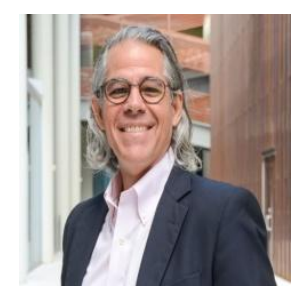

Dr. Jimmie Lenz, is the Academic Director of the Master of Engineering in Financial Technology and Master of Engineering in Cybersecurity at Duke University. He is the Frank and Irene Salerno Visiting Professor of Financial Economics. He is an experienced executive, lecturer, and scholar in the field of finance, technology, and capital markets.

His successes have propelled him into a number of senior management roles within the finance community including leading an NYSE broker dealer with foreign and domestic operations, Chief Risk Officer and Chief Credit Officer at a top three broker dealer.

Jimmie holds an undergraduate degree from the University of South Carolina, a Master of Science in Finance from Washington University in Saint Louis, and Doctor of Business Administration-Finance from Washington University's Olin Business School.

Citation: Dr. Jimmie Lenz. "Sales Practices: Monitoring Sales Activity for Anomalous Behaviors" International Journal of Managerial Studies and Research (IJMSR), vol 8, no. 11, 2020, pp. 21-25. doi: https://doi.org/10.20431/2349-0349.0811003.

Copyright: (C) 2020 Authors. This is an open-access article distributed under the terms of the Creative Commons Attribution License, which permits unrestricted use, distribution, and reproduction in any medium, provided the original author and source are credited. 